\title{
Spectral Performance of a Micromachined Infrared Spectrum Analyzer in Silicon
}

\author{
Seong Ho Kong and Reinoud F. Wolffenbuttel
}

\begin{abstract}
The spectral performance of a grating-based optical microspectrometer fabricated in silicon is presented. Fabrication is based on IC-compatible micromachining with the optoelectronic components distributed over two silicon wafers. One wafer contains an aluminum-based grating and the other an array of polysilicon thermocouples. Device dimensions are typically $5 \times 5 \times 1 \mathrm{~mm}^{3}$, with the optical path defined by an aligned wafer-to-wafer bond. The optical design constraints of this microsystem are discussed. Measurements confirm an infrared (IR) operating range between 2 and $5 \mu \mathrm{m}$ and spectral resolution $\mathbf{R}=\mathbf{1 0}$.
\end{abstract}

Index Terms-Infrared spectrum analyzer, microspectrometer, optical sensor.

\section{INTRODUCTION}

$\mathbf{S}^{\mathrm{H}}$ PECTRAL analysis of an optical spectrum is a well-established technique in physics, chemistry, and biology. Spectroscopic measurement of the emission and absorption spectra of a particular atom provides detailed information about its energy band structure, whereas analysis of a molecule shows the energies associated with the chemical bonds [1]. In chemical analysis, the fluorescence spectrum is widely used to identify the composition of a sample solution and to measure their concentrations [2]. Similarly, in chromatography the wavelengthdependent absorption of the chemical constituent between the source and the entrance slit of a spectrometer is measured in both the visible and infrared (IR) part of the spectrum [3], [4].

Available high-performance multiple-grating macroscopic spectrometers feature an impressive spectral resolution $\mathrm{R}=\lambda / \Delta \lambda$ that exceeds $\mathrm{R}=10^{6}$, where $\Delta \lambda$ denotes the $-3 \mathrm{~dB}$ power bandwidth at a particular wavelength setting $\lambda$ [5]. However, these are bulky and expensive. Such a resolution specification is required in fields such as astronomy, but often exceeds by far what is required in industrial applications where issues such as cost, sample volume, and measurement time prevail. Industrial applications typically require $100<\mathrm{R}<1000$, although color sensing is possible with $\mathrm{R}<10$. Microspectrometers are small, lightweight, and, in the case of fabrication in silicon using IC process-compatible technologies, offer the possibility for realizing intelligent optoelectronic systems on a chip by co-integration of optics with microelectronics [6].

Manuscript received June 15, 2003; revised May 24, 2004. This work was supported by the Netherlands Technology Foundation (STW) under Grant DEL55.3733.

S. H. Kong was with the Delft University of Technology, Faculty of ITS, Department for Microelectronics, 2628 CD Delft, The Netherlands. He is now with the Kyungpook National University, Taegu, South Korea.

R. F. Wolffenbuttel is with the Delft University of Technology, Faculty of ITS, Department for Microelectronics, 2628 CD Delft, The Netherlands.

Digital Object Identifier 10.1109/TIM.2004.834050

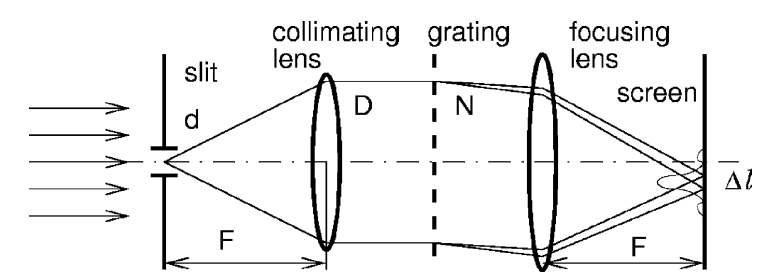

Fig. 1. Optical diagram of the basic spectrometer.

Spectrometers are basically composed of the following components:

- an input slit for spatial sampling of the radiation;

- a collimating lens (mirror) to produce a collimated light beam;

- a dispersion element (in case of a grating, it deflects the different wavelengths presented in the light beam to different angles);

- a focusing lens that produces image of the input slit in the plane of the sensor with the position of the image depending on the wavelength;

- a detector or array of detectors.

The optical path in between the lenses, used for optical signal conditioning (collimating and focusing of the incident and refracted light beam, respectively), is shown in Fig. 1.

Gratings and Fabry-Perot optical resonance cavities have been applied successfully as dispersion elements in a microspectrometer [7]-[12]. Also, the fabrication of arrays of thermoelectric detectors has been demonstrated. The dimensional and cost advantages should apply at the system level, which calls for on-chip co-integration of the optical and electronic components [6]. The IC-compatibilty of the MEMS fabrication required to achieve this co-integration of electronic and optical components introduces a tradeoff. Moreover, the optical performance of the microspectrometer depends strongly on the availability of a sufficiently long optical path, which does not combine well with minimum die area for low cost. The implementation of lenses leads to assembly at the individual die level, which would be at the expense of batch processing. In the microspectrometer presented here, the emphasis is on compatibility considerations and batch processing. As a consequence, wafer-level assembly using low-temperature silicon wafer-to-wafer bonding has been used. This results in a small, simple, and lensless configuration. As will be demonstrated, this limits spectral resolution to about $\mathrm{R}=10$. This limitation is more than compensated for in the restricted range of moderately demanding applications by the advantages of small size and low cost in batch fabrication. Spacers and assembly at the 


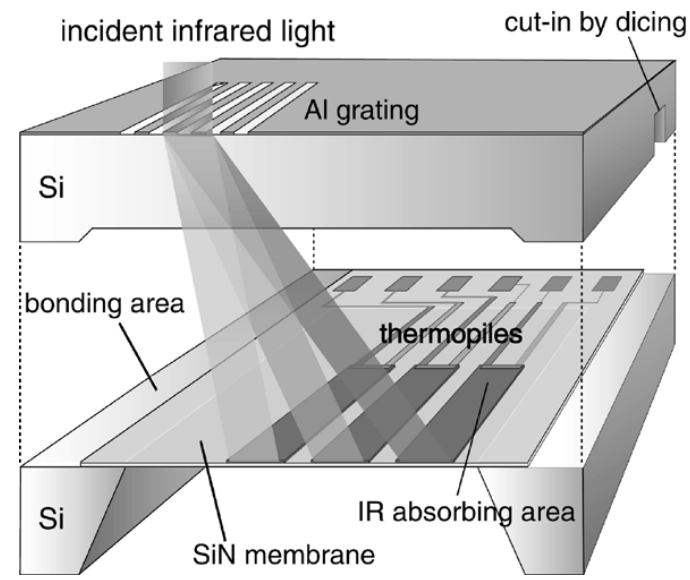

Fig. 2. Cross-sectional schematic view of the IR spectrometer structure.

individual chip level would be required to yield a device that serves the vast majority of industrial applications.

\section{DeVICE Operation}

Silicon is highly transparent for wavelengths, exceeding $1.1 \mu \mathrm{m}$, beyond which free-carrier absorption can be disregarded. Therefore, bulk silicon can be used to define the optical path rather than air. Aluminum, which is conventionally exclusively used for interconnect, is also applied here for fabrication of the grating and for shielding the array of detectors to prevent front-side illumination by the incident light. Aluminum is used for fabrication of the grating, despite the fact that the reflectance is lower than that of silver or gold in the major part of the visible and near infrared spectral range, because it is a more acceptable material in an IC fabrication facility [6].

The IR spectrometer, shown schematically in Fig. 2, consists of two independently processed wafers which are bonded in the final step [10]. A two-wafer approach is taken, with the grating fabricated in the upper wafer and the thermocouple-based infrared detector array in the lower wafer. This approach facilitates fabrication of detector and readout circuits in the second wafer without any concern over a possible process compatibility infringement.

Impinging light is dispersed in the grating, which is composed of 30 or 60 slits, with a grating constant ranging from 4 to $20 \mu \mathrm{m}$. The length of each of the strips is $400 \mu \mathrm{m}$. The light travels through the upper wafer and is projected onto an array of thermocouples. Only the hot junction is exposed; thus, IR optical power absorbed at a particular element leads to a localized temperature difference that is detected in the thermocouple. The width of a single IR detector on the bottom wafer is $100 \mu \mathrm{m}$. The number of detectors in one array ranges between six and 16 and depends on the detectable wavelength range, which is, in turn, determined by the grating constant.

The thermocouples are $\mathrm{p}$ - and n-type polysilicon layers with aluminum interconnect to short-circuit the junctions to avoid a p-n forward voltage drop. No special IR absorbing materials were deposited. The absorption depends solely on the optical properties of the dielectric layers that result from IC-processing,

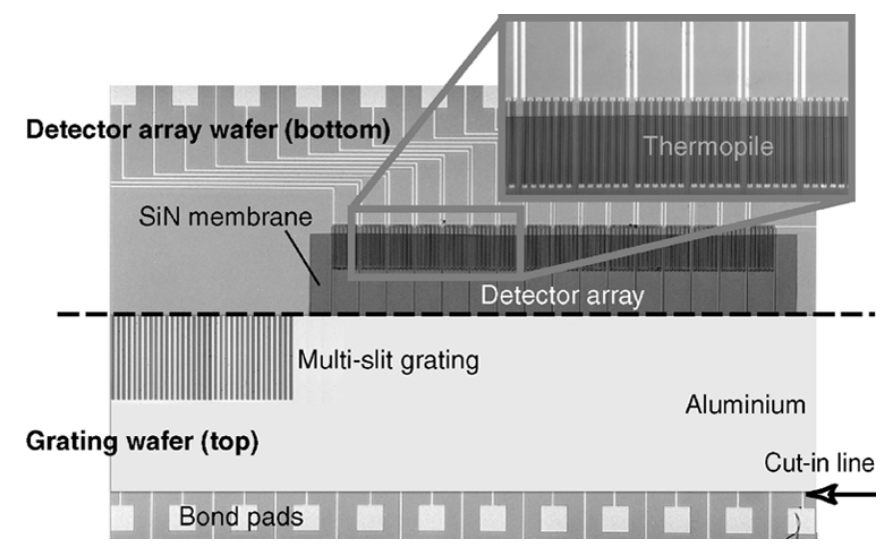

Fig. 3. Composite photograph of the fabricated microspectromter.

which introduces a wavelength dependence of the spectral response in the thermopile thermal detector. Fig. 3 shows the top view of a fabricated device.

\section{Performance Limitations}

In principle, the spectral resolution in a grating-based spectrometer, when using the first-order diffraction spectrum, should be equal to the number of slits $\mathrm{N}$ in the grating: $\mathrm{R}=\mathrm{N}$ [13]. In theory, there is no limit to the number of elements in the grating and, thus, to the resolution. However, this assumes Fraunhofer rather than Fresnel diffraction. For ensuring Fraunhofer diffraction (the laws of far field optics to be valid), the wavefront of both the radiation impinging on the grating and the detector should be planar. This implies that the distance between the entrance slit of the spectrometer and the grating, as well as the distance between the grating (which can within this context also be considered a single slit), and the surface onto which the dispersed spectrum is projected (i.e., the detector), should be "large enough." This condition is more quantitatively expressed as the Rayleigh distance $\mathrm{D}=\mathrm{w}^{2} / \lambda$, where $\mathrm{w}$ denotes the slit width and $\lambda$ the wavelength of the light incident on the slit.

The case of an N-element grating with a pitch $\mathrm{p}$ (a regular pattern of $\mathrm{p} / 2$-wide aluminum strips spaced at $\mathrm{p} / 2$ ) results in a "slit" of width $\mathrm{w}=\mathrm{N} \cdot \mathrm{p}$. The resulting Rayleigh distance at wavelength $\lambda$ is equal to $\mathrm{D}=\mathrm{N}^{2} \mathrm{p}^{2} / \lambda$. At this stage, the consequences of the self-imposed dimensional constraints become apparent. The wafer bonding limits the length of optical path available to the wafer thickness $t_{\mathrm{w}}=525 \mu \mathrm{m}$. This results in a maximum useable number of elements in the grating $\mathrm{N}_{\max }^{2}=$ $t_{\mathrm{w}} \cdot \lambda / \mathrm{p}^{2}$. The pitch is limited by technological constraints and by the diffraction angle that results from the maximum wavelength to be analyzed. Using $\mathrm{p}=4 \mu \mathrm{m}$ and $\lambda=4 \mu \mathrm{m}$ yields $R_{\max }=\mathrm{N}_{\max }=12$.

\section{MEASUREMENTS}

The grating has been designed for a gap half the pitch for maximum suppression of the second-order spectrum in order to maximize the free spectral range when using a detector array aligned with the first-order diffraction spectrum. Fig. 4 shows the presence of optical power at $\mathrm{m}=2$, which demonstrates that special care is required to achieve this. The cause is the 


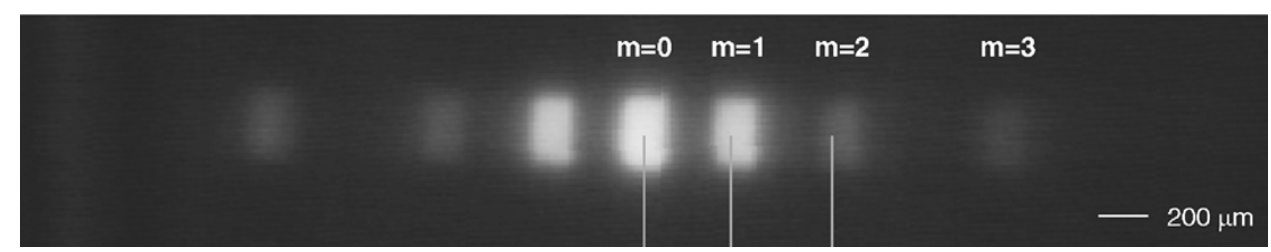

Fig. 4. CCD recording of the diffraction pattern of a 60 -element 4- $\mu \mathrm{m}$-pitch grating fabricated using standard interconnect patterning.

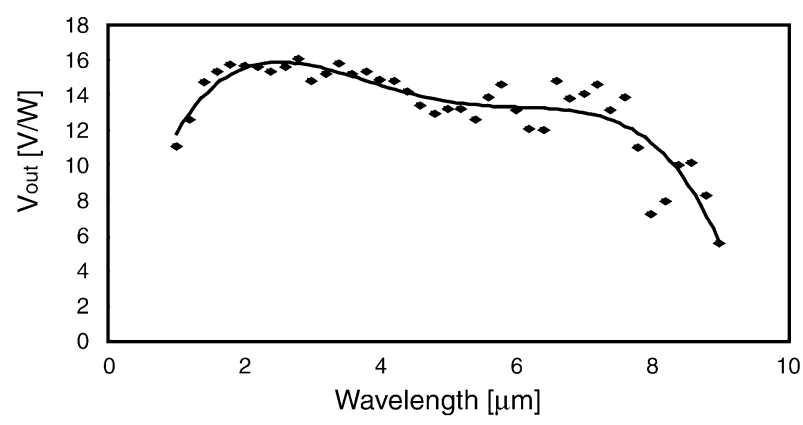

Fig. 5. Spectral response of a poly-Si thermopile IR detector.

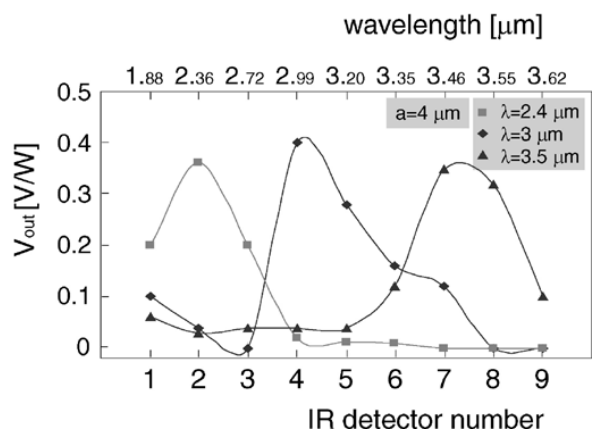

Fig. 6. Spectral response of the IR microspectrometer with grating constant at $4 \mu \mathrm{m}$ when illuminated using a monochromator set at $\lambda=2.4,3$, and $3.5 \mu \mathrm{m}$.

common practice in IC technology to overetch the aluminum. The standard etching procedure has been optimized for electrical constraints (avoiding short-circuits) and is not necessarily optimum for optical components. This indicates that even this highly compatible process step needs to be analyzed critically. Since the grating metal is on a separate wafer and needs not be combined with electrical circuit interconnect, the problem can be easily solved. A slightly modified IC-compatible metal etch yields an adequate suppression of the second-order spectrum.

The spectral uniformity of the thermocouples has been performed using a blackbody source illuminating a monochromator. A computer-controlled measurement setup has been used to tune the monochromator through the spectrum. The output was used to illuminate both the device under test and a reference detector. The thermocouples have been used without an absorbing coating. Basically, silicon-oxide and silicon-nitride layers have been used for this purpose. This approach circumvents any fabrication compatibility infringement and, as is demonstrated by the response of a thermopile composed of 32 thermoelectric elements shown in Fig. 5, the results are acceptable in many applications. Since the spectrum is already dispersed, the correction for the wavelength-dependent absorption is determined by the detector position and can easily be implemented.

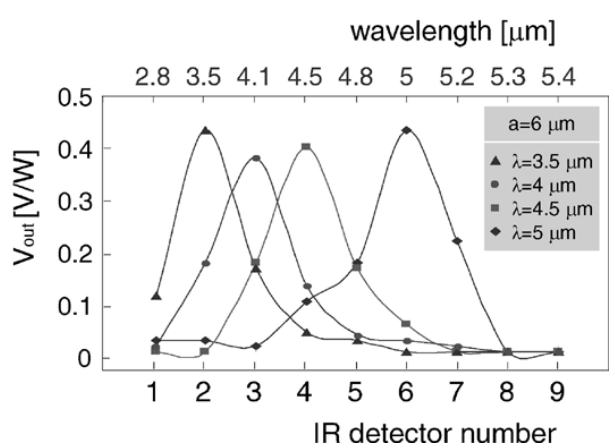

Fig. 7. Spectral response of the IR microspectrometer with grating constant at $6 \mu \mathrm{m}$ when illuminated using a monochromator set at $\lambda=3.5,4,4.5$, and $5 \mu \mathrm{m}$.

The spectral selectivity has been measured for two grating constants $(4$ and $6 \mu \mathrm{m})$ and the same blackbody source set at different temperatures, and the results are shown in Figs. 6 and 7. The response peaks at a larger detector number in case of longer wavelength illumination. The half-power spectral bandwidth at $\lambda=4 \mu \mathrm{m}$ is about $0.4 \mu \mathrm{m}$; thus, $\mathrm{R} \approx 10$.

\section{CONCLUSION}

The measured performance, as shown in Fig. 7, is in agreement with this theoretical limit. It would be pointless to increase the number of elements in the grating at the given device dimensions. An improved resolution is feasible only by having a larger $\mathrm{N}$ associated with an enlarged optical path length between grating and detector array. This can be achieved by the stacking of wafers or implementing spacers. Also, the pitch can be further reduced. However, the main impediment that limits the spectral resolution of a fully integrated silicon optical microspectrometer of the type presented here is the definition of a sufficiently long optical path and the fact that a lensless configuration is used. Implementation of lenses or deformed mirrors would give a significantly improved optical performance. However, batch processing would be impaired.

\section{ACKNOWLEDGMENT}

The authors are indebted to DIMES-tc for assistance in the fabrication of devices.

\section{REFERENCES}

[1] B. Stuart, Modern IR Spectroscopy. New York: Wiley, 1996.

[2] V. Iordanov, G. Lubking, R. Ishihara, R. F. Wolffenbuttel, P. M. Sarro, and M. J. Vellekoop, "Silicon thin-film UV filter for NADH analysis," Sens. Actuators, vol. A97-98, pp. 161-166, 2002. 
[3] D. Rossberg, "Silicon micromachined infrared sensor with tuneable wavelength selectivity for application in infrared spectroscopy," Sensors Actuators, vol. A 46-47, pp. 413-416, 1995.

[4] G. Minas, J. S. Martins, C. Pereira, C. Lima, R. F. Wolffenbuttel, and J. H. Correia, "Lab-on-a-chip for measuring uric acid in biological fluids," in Proc. Eurosensors XVI, Prague, Czech Rep., Sep. 15-18, 2002, pp. 66-69.

[5] P. Lindblom, M. Meinander, and T. Olsson, "Spectroscopy with the MEGA spectrometer, A very high resolution grating spectrometer," Rev. Sci. Instrum., vol. 61, pp. 2546-2548, 1990.

[6] R. F. Wolffenbuttel, Ed., Silicon Sensors and Circuits: On-Chip Compatibility. London, U.K.: Chapman \& Hall, 1996.

[7] R. F. Wolffenbuttel, "Silicon photodetectors with a selective spectral response," in Sensors Update, H. Baltes, J. Hesse, and J. Korvink, Eds. New York: Wiley-VCH, 2001, vol. 9, pp. 69-101.

[8] T. A. Kwa and R. F. Wolffenbuttel, "Integrated grating/detector array fabricated in silicon using micromachining techniques," Sensors Actuators, vol. A31, pp. 259-266, 1992.

[9] G. M. Yee, N. I. Maluf, P. A. Hing, M. Albin, and G. T. A. Kovacs, "Miniature spectrometers for biochemical analysis," Sensors Actuators, vol. A58, pp. 61-66, 1997.

[10] S. H. Kong, D. D. L. Wijngaards, and R. F. Wolffenbuttel, "Infrared micro-spectrometer based on a diffraction grating," Sens. Actuators, vol. A92, pp. 88-95, 2001.

[11] J. H. Correia, M. Bartek, and R. F. Wolffenbuttel, "High-selectivity single-chip spectrometer in silicon for operation in the visible part of the spectrum," IEEE Trans. Electron Devices, vol. 47, pp. 553-559, Mar. 2000.

[12] J. H. Jerman, D. J. Clift, and S. R. Mallinson, "A miniature Fabry-Perot interferometer with a corrugated silicon diaphragm support," Sensors Actuators, vol. A29, pp. 151-158, 1991.

[13] M. Born and E. Wolf, Principles of Optics, fifth ed. New York: Pergamon, 1975.

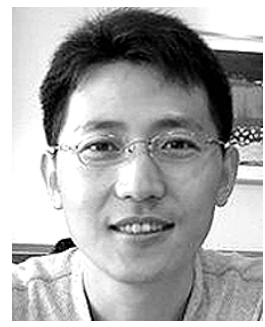

Seong Ho Kong was born in Youngcheon, South Korea, in 1967. He received the B.S. degree in electrical engineering From Kyungpook National University, Taegu, South Korea, in 1993, the M.S degree in mechatronics and precision engineering from Tohoku University, Sendai, Japan, in 1996, and the Ph.D. degree from Delft University of Technology, Delft, The Netherlands, in 2002.

From 2002 to 2003, he was with ASM Europe, Bilthoven, The Netherlands. He is presently with the Kyungpook National University.

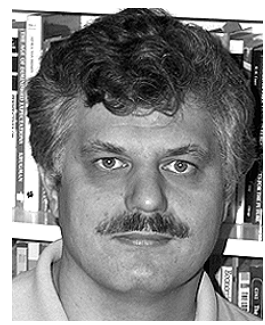

Reinoud F. Wolffenbuttel received the M.Sc degree in 1984 and the Ph.D. degree in 1988, both from the Delft University of Technology, Delft, The Netherlands.

From 1986 to 1993, he was an Assistant Professor, and since 1993, he has been an Associate Professor at the Department of Microelectronics, Faculty of Information Technology and Systems, Delft University of Technology. He is involved in instrumentation and measurement, in general, and on-chip functional integration of microelectronic circuits and silicon sensor,

IC process compatible MEMS fabrication, and silicon microsystems, in particular. He was a visitor at the University of Michigan, Ann Arbor, MI, in 1992, 1999, and 2001, Tohoku University, Sendai, Japan, in 1995, and The Swiss Federal Institute of Technology, Lausanne, Switzerland, in 1997.

$\mathrm{He}$ is the recipient of the 1997 NWO pioneer award. He served as General Chairman of the Dutch National Sensor Conference in 1996, Eurosensors in 1999, and the MicroMechanics Europe workshop in 2003. 